\title{
Ely A. Wolin: Top 3 Differentials in Nuclear Medicine: A Case Review
}

\section{Thieme Medical Publishers Inc., 2019, (ISBN: 978-1-626-23344-7)}

\author{
Luigi Mansi ${ }^{1}$
}

Published online: 31 August 2019

(C) Springer-Verlag GmbH Germany, part of Springer Nature 2019

This is a didactic book, having as main goal the preparation for the examination in nuclear medicine and/or radiology. It is part of the successful series Top 3 Differentials in Radiology: A Case Review, edited by William T. O'Brien, professor at the University of Cincinnati. The author of this text, concerning Nuclear Medicine, is the Lieutenant Colonel of the United States Air Force Ely A. Wolin, Program Director of the Diagnostic Radiology Residency and Assistant Professor at the Uniformed Services University of the Health Sciences in Bethesda, MD, USA.

In his editorial mission, he has been accompanied by experts working in Academy or in the Army. This is an interesting complementary choice, because military nuclear physicians or radiologists should be mainly educated to find the fastest answer, following therefore the simplest diagnostic course. Conversely, physicians working in universities have to consider also the rarest hypotheses, recruiting frequently, as second-line diagnostic frontier, a large series of clinical cases, including the most difficult and/or infrequent. Therefore, the book results in a useful synthesis that can be helpful not only for students but also for practitioners who want to learn (or refresh) how to act in a routine condition, in which the time can be a relevant issue to define the clinical usefulness. Interestingly, as author is also involved, a resident, Dr. Cathy Zhou, probably contributed as a critical judge of the quality of the text from the student's point of view.

The book of 338 pages is enriched by 267 illustrations and also includes a complimentary access to a digital copy on

Luigi Mansi

mansi.luigi@libero.it

1 Interuniversity Research Center for Sustainability (CIRPS), Naples, Italy https://medone.thieme.com. High-quality images regard 147 carefully selected clinical cases, providing illustrative examples constructed across all imaging modalities, which have the capability to deliver a robust, well-rounded nuclear medicine preparation. Of course, in my opinion, deriving from the over 40 years of academic experience, this book, effective for passing an exam, has to be integrated by more extensive and/or specific text, also including a wider deepening of basic, pathophysiological, and clinical premises.

The publication has a simple didactic structure, being structured in 12 chapters. The first 8 are organized on the basis of the most important clinical issues, i.e., neuro, thyroid and parathyroid, cardiac, lung, hepatobiliary, gastrointestinal, genitourinary, and bone imaging. In the following chapters, cases regarding infection and inflammation (9), PET-FDG (10), tumor not PET, i.e., studied using SPECT (11), are presented. The twelfth part regards quality control, an important issue that has to be known either for the examination or in the clinical practice. The index of differential diagnoses and the index of the key findings are also included at the end of the book.

Each case is formatted as a two-page unit, with the left featuring clinical images, succinctly captioned findings, and pertinent clinical history. At the right, key imaging gamut, additional diagnostic considerations, and clinical pearls are listed to arrive to the final editorial goal: how to make a differential diagnosis rankordered by the "Top 3."

I suggest this publication either to trainees or to practitioners who want to learn or improve their capability to obtain a differential diagnosis based on nuclear medicine. The book can also be useful in libraries associated to nuclear medicine departments, resulting in easy availability as practical contribution to the everyday work. 